\title{
Catalyzing a Research Agenda for Enhancing Engineering Education through Institutional Collaborations
}

\section{Dr. Keith W. Buffinton, Bucknell University}

Keith W. Buffinton is a Professor of Mechanical Engineering and former Dean of the College of Engineering at Bucknell University. He received his B.S. in Mechanical Engineering from Tufts and his M.S. and Ph.D. from Stanford. Following his graduate studies, he worked as a post-doctoral researcher in the Institute for Mechanics at the Swiss Federal Institute of Technology in Zurich, Switzerland. From 2001 through 2004 he served as co-director of Bucknell's Institute for Leadership in Technology and Management and from 2003 through 2007 as Associate Dean of the College of Engineering. In 2003 he received Bucknell's Christian R. and Mary F. Lindback Award for Distinguished Teaching.

Prof. Buffinton's scholarly interests range across the areas of multibody dynamics, nonlinear control, mechanical design, systems thinking, entrepreneurship, engineering management education, and his primary research focus, the dynamics and control of robotic systems. He has been the recipient of external grants from a number of funding agencies including the National Science Foundation, the Office of Naval Research, the Ben Franklin Technology Center of Pennsylvania, and most recently the Kern Family Foundation. As Dean of Engineering, Prof. Buffinton particularly sought to enhance support for students from under-resourced backgrounds as well as to promote the creation of an Ecology of Entrepreneurship. Prof. Buffinton has been a past member of the Executive Board of the ASEE Engineering Deans Council and was formerly Co-Chair of the ASEE EDC Undergraduate Experience Committee.

\section{Dr. Vincent P. Manno, Franklin W. Olin College of Engineering}

VINCENT P. MANNO is Provost and Dean of Faculty, Professor of Engineering at the Franklin W. Olin College of Engineering. He received a BS from Columbia University and MS and Sc.D. from M.I.T. His field of expertise is thermal-fluid dynamics with applications in power, electronics thermal management, and manufacturing. He has authored more than 140 technical publications. His honors include SAE's Teetor Award, Rosten Award for Thermal Analysis of Electronic Equipment, ASME Curriculum Innovation Award, and Fischer Engineering Teacher of the Year Award. He is an ASME Fellow and on the Board of Directors of ASEE's Engineering Research Council.

\section{Dr. Joseph J. Helble, Dartmouth College}

Joseph J. Helble is Professor of Engineering, and Dean of the Thayer School of Engineering at Dartmouth College, a position he has held since 2005. Prior to Dartmouth, Dr. Helble was the AAAS Revelle Fellow, spending a year on staff in the U.S. Senate with a focus on science policy. Previously, he was Professor and Chair of Chemical Engineering at the University of Connecticut, and from 1987 to 1995, a researcher at Physical Sciences Inc. Dr. Helble is the author of over 100 publications and 3 U.S. patents, the recipient of several awards for his scholarly work, and a 2014 co-recipient of the National Academy of Engineering Gordon Prize for the development of Dartmouth's Engineering Entrepreneurship Program. He presently serves as Chair of the ASEE Engineering Deans Public Policy Committee. Dr. Helble holds a B.S from Lehigh University and a Ph.D. from MIT.

\section{Dr. Susan M Lord, University of San Diego}

Susan M. Lord received a B.S. from Cornell University and the M.S. and Ph.D. from Stanford University. She is currently Professor and Chair of Electrical Engineering at the University of San Diego. Her teaching and research interests include electronics, optoelectronics, materials science, first year engineering courses, feminist and liberative pedagogies, engineering student persistence, and student autonomy. Her research has been sponsored by the National Science Foundation (NSF). Dr. Lord is a fellow of the ASEE and IEEE and is active in the engineering education community including serving as General Co-Chair of the 2006 Frontiers in Education (FIE) Conference, on the FIE Steering Committee, and as President of the IEEE Education Society for 2009-2010. She is an Associate Editor of the IEEE Transactions on Education. She and her coauthors were awarded the 2011 Wickenden Award for the best paper in the Journal 
of Engineering Education and the 2011 Best Paper Award for the IEEE Transactions on Education. In Spring 2012, Dr. Lord spent a sabbatical at Southeast University in Nanjing, China teaching and doing research.

\section{Dr. Ann F. McKenna, Arizona State University, Polytechnic campus}

Ann F. McKenna is a Professor in the Ira A. Fulton Schools of Engineering and Director of The Polytechnic School at Arizona State University. Prior to joining ASU she served as a program director at the National Science Foundation in the Division of Undergraduate Education, and was on the faculty in the Department of Mechanical Engineering and Segal Design Institute at Northwestern University. Dr. McKenna received her B.S. and M.S. degrees in Mechanical Engineering from Drexel University and $\mathrm{Ph} . \mathrm{D}$. from the University of California at Berkeley.

\section{Dr. Matthew W. Ohland, Purdue University, West Lafayette (College of Engineering)}

Matthew W. Ohland is Professor of Engineering Education at Purdue University. He has degrees from Swarthmore College, Rensselaer Polytechnic Institute, and the University of Florida. His research includes the longitudinal study of engineering students, team assignment, peer evaluation, and active and collaborative teaching methods and his team received Best Paper awards from the Journal of Engineering Education in 2008 and 2011 and from the IEEE Transactions on Education in 2011 and 2015. Dr. Ohland is an ABET Program Evaluator for ASEE and was the 2002-2006 President of Tau Beta Pi. He is a Fellow of the ASEE and IEEE. 


\title{
Catalyzing a Research Agenda for Enhancing Engineering Education through Institutional Collaborations
}

\begin{abstract}
To augment the extensive engineering education research that has been done over the past decades, greater opportunities are needed for institutional leaders and education practitioners to directly share the pedagogical practices that have worked best (and perhaps not so well) at their institutions as well as to explicitly consider bi-directional scalability and adaptability between institutions. The National Science Foundation (NSF) funded workshop described here brought together a small group of thought leaders from various institutions to share their ideas and experiences and to develop a research agenda for creating productive collaborations among a wide range of institutions of different types, characteristics, and missions. The workshop sought to develop a framework for how diverse institutions can effectively impact engineering education in addressing questions such as: How to scale, adapt, and transfer best practices? What are the roles of differing types of institutions in engineering education research and innovation? How can engineering education research at all institutions be enhanced? Preliminary results presented in this paper include quantitative measures of the characteristics of the participating institutions, the results of a pre-workshop survey completed by each institution about educational constraints and opportunities, and highlights of the workshop itself. The workshop brought to light the significant changes that have already occurred in engineering education in recent years, the need to focus on cultural change rather than content change, and the recognition that institutions of higher learning, the NSF, and engineering professional societies have not yet succeeded in creating the right culture, climate, and educational infrastructure for fully implementing change in engineering education based on the most effective approaches to engaging students. Based on the outcomes of the workshop, engineering education must clearly change in ways that allow it to focus on defining problems differently and to facilitate a shift in both the mindsets of faculty and the mindsets they cultivate in students.
\end{abstract}

\section{Introduction}

In recent years, considerable research has been done on effective engineering pedagogy. The research has shown that while passive knowledge acquisition and assimilation (e.g. traditional lecturers) can be effective for a fraction of learners, exclusive utilization of such approaches yields limited learning outcomes [1-5]. Broad based active approaches to learning, using, for example, Problem-Based Learning, Project-Based Learning, POGIL (Process Oriented Guided Inquiry Learning), Collaborative Learning, Flipped Classrooms, as well as techniques as simple as Think-Pair-Share and Journaling, can be more effective in promoting learning for a broader spectrum of students not only in the classroom but also throughout an engineering graduate's career [6-11]. The emphases within engineering education have also shifted as the need for broader engagement with diverse populations of learners has been recognized and industry has expressed a desire for more engineering employees who bring not only technical skills to their work but also a broad entrepreneurial mindset with a clear understanding of the societal underpinnings of engineering decision making and the need to address diversity [12-15]. Additionally, the ways in which students can be engaged through the use of online educational 
resources, and the corresponding use of online tools to assess student learning, have changed the ways in which students can access educational opportunities as well as dramatically altered costof-delivery models of education [16].

More broadly, data from industry suggests, and have been supported by recent Gallup surveys, that the attitudes, behaviors, and motivations (what one might call "mindsets") of engineering graduates are increasingly important to their future careers. Unfortunately, this shift in expectations occurring in industry at companies such as Microsoft, Boeing, and Google has largely not yet been recognized within institutions of higher education. Indeed, according to a 2013 Gallup poll conducted on behalf of the Lumina Foundation, 96\% of chief academic officers believe their institutions are "very/somewhat effective at preparing students for the world of work," while only $11 \%$ of business leaders "strongly agree that graduating students have the skills and competencies that their businesses need.” Moreover, only 14\% of the general public "strongly agrees that college graduates in this country are well prepared for success in the workforce" [17]. These observations are extremely important considerations when looking to the future of engineering education and making it appropriately relevant to students, industry, and the needs of society.

How best to take advantage of evidence based research and the most effective approaches to engineering education is institution dependent, faculty dependent, and course dependent. A better understanding of best practices in engineering education across the spectrum of engineering programs is thus necessary. This is particularly timely as large institutions look to restructure their curricula to respond to increasingly tight economic constraints and demands for greater student accessibility. Small institutions are likewise currently reconsidering the longterm viability of high-cost residential learning and changing models of credentialing as well as industrial and societal expectations.

Opportunities for leaders within various institutions to directly share the practices that have worked best (and perhaps not so well) are lacking. Institutions also have insufficient opportunities to explicitly consider bi-directional scalability and adaptability between institutions that share a common goal of educating effective engineers but experience different constraints. The NSF-funded workshop described here brought together a small group of thought leaders from various institutions to share their ideas and experiences and to develop a research agenda for creating productive collaborations among a wide range of institutions of different types, characteristics, and missions. The workshop sought to develop a viable research agenda that can frame how diverse institutions can effectively impact engineering education in addressing questions such as: How to scale, adapt, and transfer best practices? What are the roles of differing types of institutions in engineering education research and innovation? How can engineering education research at all institutions be enhanced? This work builds on previous examples of the cross-fertilization that can occur, such as that demonstrated in the work of Dr. Richard Layton at Rose-Hulman Institute of Technology who based practices for managing growing enrollments in Mechanical Engineering at Rose-Hulman on approaches used at Purdue University to implement student-centered pedagogies in large-enrollment classes [18]. 


\section{Participating Institutions and Axes of Variation}

The spring 2017 workshop that is the focus of this paper invited faculty and administrative representatives from 16 institutions ranging from small, predominantly undergraduate institutions through large, research oriented institutions. The specific institutions that were invited and agreed to participate were:

- Arizona State University (ASU), Tempe, Arizona

- Bucknell University, Lewisburg, Pennsylvania

- Dartmouth College, Hanover, New Hampshire

- Harvey Mudd College, Claremont, California

- Ohio State University, Columbus, Ohio

- Franklin W. Olin College of Engineering, Needham, Massachusetts

- Oregon State University, Corvallis, Oregon

- Purdue University, West Lafayette, Indiana

- Rose-Hulman Institute of Technology, Terre Haute, Indiana

- Rowan University, Glassboro, New Jersey

- Stanford University, Palo Alto, California

- Tufts University, Medford, Massachusetts

- University of San Diego (USD), San Diego, California

- University of Texas at El Paso, El Paso, Texas

- Western Kentucky University, Bowling Green, Kentucky

- Worcester Polytechnic Institute (WPI), Worcester, Massachusetts

These institutions were selected because of the range of characteristics they represented along 10 different "axes." Based on publicly available data [19-21], the 10 axes and grouping of institutions along each axis are given below:

\section{1) Undergraduate Engineering Enrollment}

High (over 4,000) -- ASU, Ohio State, Oregon State, Purdue

Medium $(2,000$ to 4,000) -- Rose-Hulman, Stanford, U. Texas-EP, WPI

Low (under 2,000) -- Bucknell, Dartmouth, Harvey Mudd, Olin, Rowan, Tufts, USD, W. Kentucky

2) Percent Female Undergraduate Engineering Enrollment

High (over 40\%) -- Harvey Mudd, Olin

Medium (20\% to 40\%) -- Bucknell, Dartmouth, Ohio State, Purdue, Rose-Hulman, Stanford, Tufts, USD, U. Texas-EP, WPI

Low (under 20\%) -- ASU, Oregon State, Rowan, W. Kentucky

3) Percent Other Underrepresented Undergraduate Engineering Enrollment

High (over 40\%) -- Harvey Mudd, Olin

Medium (20\% to 40\%) -- Bucknell, Dartmouth, Ohio State, Purdue, Rose-Hulman, Stanford, Tufts, USD, U. Texas-EP, WPI

4) Public vs. Private

Public -- ASU, Ohio State, Oregon State, Purdue, Rowan, U. Texas-EP, W. Kentucky

Private -- Bucknell, Dartmouth, Harvey Mudd, Olin, Rose-Hulman, Stanford, Tufts, USD, WPI 


\section{5) Engineering Undergraduates / Teaching Faculty}

High (over 25) -- ASU, Harvey Mudd, Oregon State, U. Texas-EP, W. Kentucky

Medium (15 to 25) -- Ohio State, Purdue, Rose-Hulman, WPI

Low (under 15) -- Bucknell, Dartmouth, Olin, Rowan, Stanford, Tufts, USD

6) Comprehensive Fee (Out of State for Publics)

High (over \$60,000) -- Bucknell, Dartmouth, Harvey Mudd, Olin, Stanford, Tufts

Medium $(\$ 40,000$ to $\$ 60,000)$-- Ohio State, Oregon State, Purdue, Rose-Hulman, USD, WPI

Low (under \$40,000) -- ASU, Rowan, U. Texas-EP, W. Kentucky

\section{7) Percent Engineering Applicants Admitted}

High (over 50\%) -- Oregon State, Purdue, Rose-Hulman, Rowan, U. Texas-EP, W. Kentucky, WPI

Medium (20\% to 50\%) -- ASU, Ohio State

Low (under 20\%) -- Bucknell, Dartmouth, Olin, Stanford, Tufts

\section{8) Combined SAT (MATH+CR) Mid Range}

High (over 1400) -- Olin, Stanford, Tufts

Medium (1200 to 1400) -- ASU, Bucknell, Ohio State, Purdue, Rose-Hulman, Rowan, USD, WPI

Low (under 1200) -- Oregon State, U. Texas-EP, W. Kentucky

9) Endowment / Undergraduate Student

High (over \$500k) -- Dartmouth, Olin, Rose-Hulman, Stanford

Medium (\$100k to \$500K) -- Bucknell, Harvey Mudd, Tufts, WPI

Low (under \$100k) -- ASU, Ohio State, Oregon State, Purdue, Rowan, USD, U. Texas-EP, W. Kentucky

10) 4-Year Institutional Graduation Rate

High (over 80\%) -- Bucknell, Dartmouth, Harvey Mudd, Olin, Tufts

Medium (50\% to 80\%) -- Ohio State, Rose-Hulman, Stanford, USD, WPI

Low (under 50\%) -- ASU, Oregon State, Purdue, Rowan, U. Texas-EP, W. Kentucky

The groupings for "High," "Medium," and "Low" were selected arbitrarily but do provide an indication of the range of institutions involved in the workshop. Examples of the full range of data are shown in Fig. 1. Figure 1(a) gives an indication of the overall size of the undergraduate engineering program at each of the participating institutions, with Arizona State University having the largest total number of engineering undergraduates with 11,572 (\#1 in the country according to [19]) and Olin College of Engineering having the fewest at 337. Figure 1(b) provides insights into the number of undergraduate engineering students per teaching faculty (both tenure track and non-tenure track). Western Kentucky University has 46.3 students per teaching faculty member and both Tufts University and Olin College have only 6.0.

An indication of the resources available per student at each of the institutions is shown in Fig. 1(c), which is a graph of endowment per total number of undergraduate students. The figure shows a range from $\$ 3.1 \mathrm{M}$ per student at Stanford down to $\$ 92$ per student at Western Kentucky University. Obviously, there is a wide range (4 orders of magnitude) along this particular axis. The variation in the 4-year institutional graduation rate (not just in engineering) is shown in Fig. 1(d), which presents data ranging from $88 \%$ at Dartmouth College and Tufts University to $12 \%$ at the University of Texas, El Paso. 


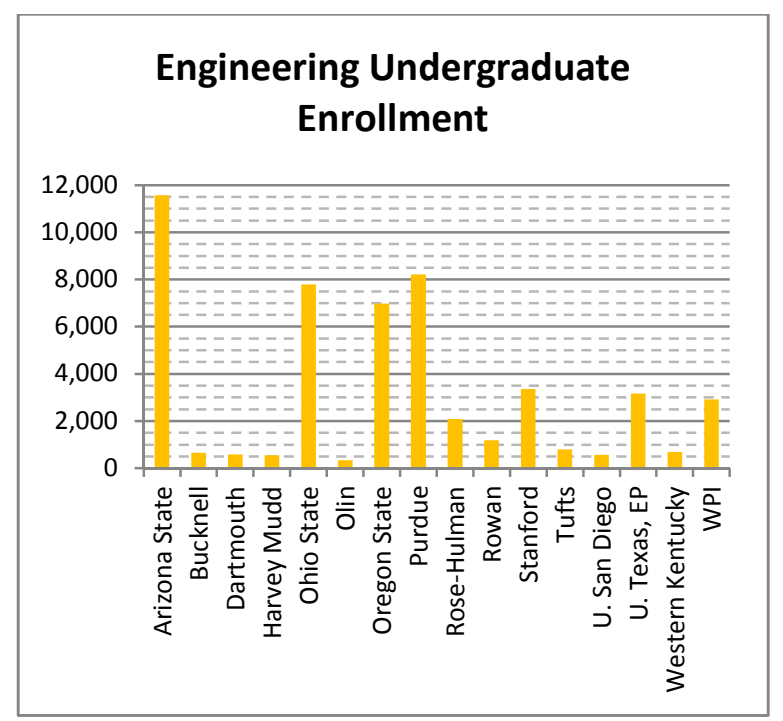

(a)

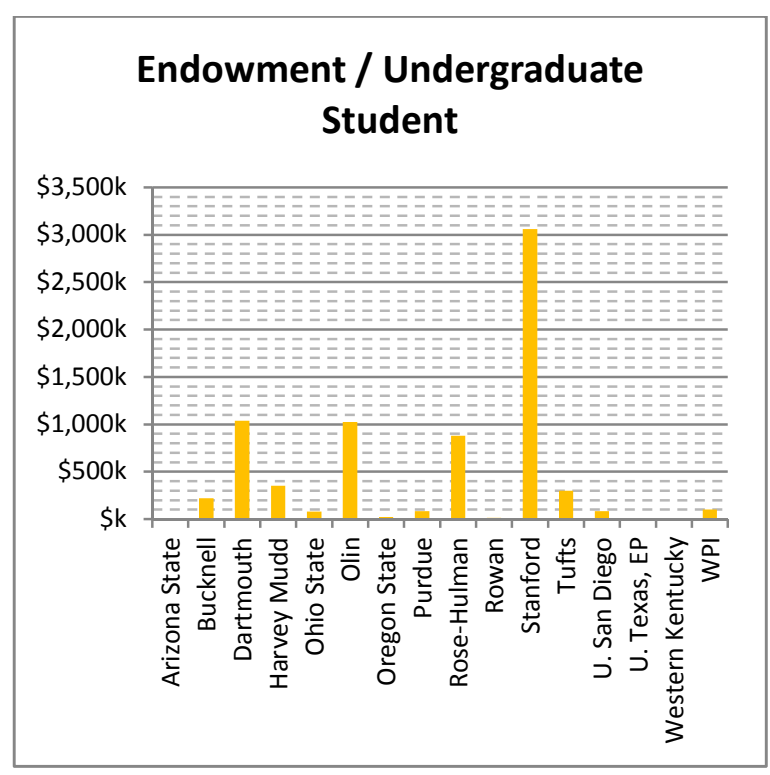

(c)

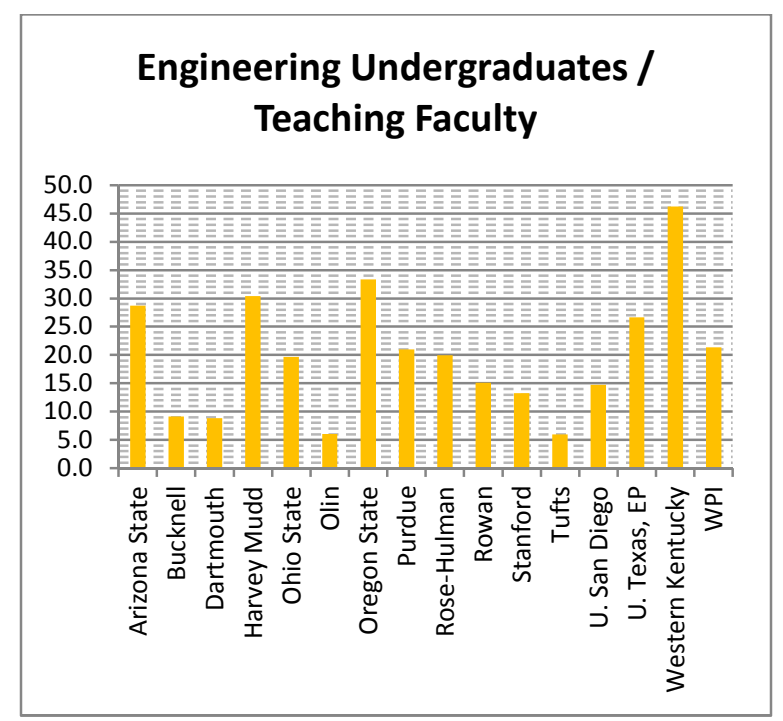

(b)

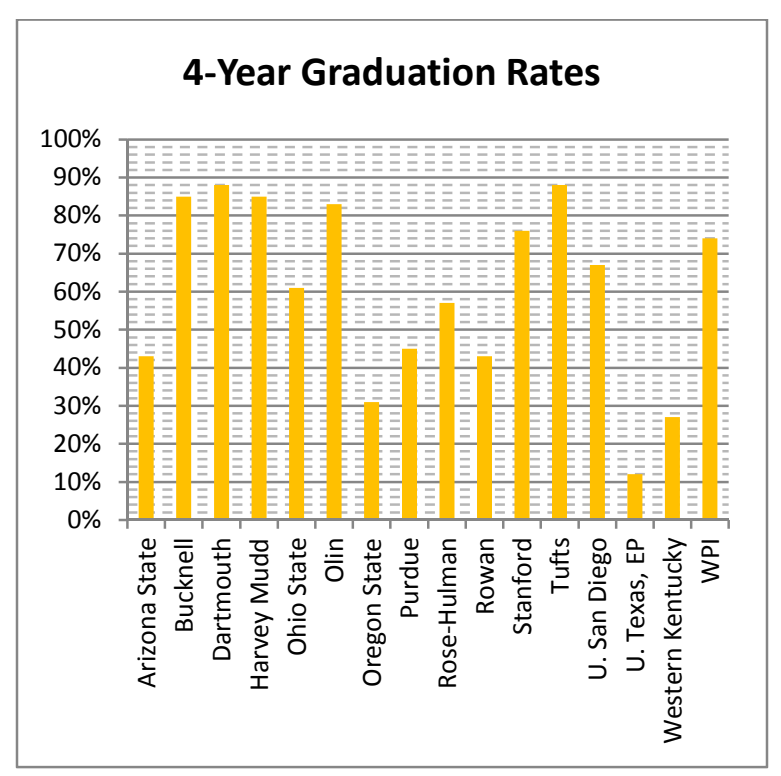

(d)

Figure 1. (a) Engineering Undergraduate Enrollment; (b) Engineering Undergraduates per Teaching Faculty; (c) Endowment per Undergraduate Student; (d) Four-Year Graduation Rates

Attempts were made to include in the workshop a broader spectrum of institutions that predominantly serve underrepresented populations, but we were unfortunately not successful in attracting as wide a range of institutions as desired. The percentage of underrepresented students (other than female) at the participating institutions was generally less than $25 \%$, with the exception of the University of Texas, El Paso which serves an 80\% Hispanic population. Based on data recently available through The New York Times from The Equality of Opportunity Project on access to college across income distribution [23], the range of socio-economic diversity among participating institutions was also limited. Only two of the 16 participating institutions (University of Texas, El Paso and Western Kentucky University) draw more than 
$10 \%$ of their student population from households with incomes in the bottom $20 \%$ of U.S. families (less than $\$ 20,000$ per year). The University of Texas, El Paso and Western Kentucky University were also the only two institutions with median family incomes of less than $\$ 100,000$ (\$37,500 and $\$ 71,300$, respectively).

\section{Survey of Participating Institutions}

While there is a wide range in the quantitative measures associated with the participating institutions, they do not tell the full stories of the characters and priorities of these institutions nor the particular challenges they face. To delve more deeply into the experiences and aspirations of the participating institutions, we developed a short survey to be completed at each institution. Since we specifically structured the workshop to bring together faculty actively participating in classroom teaching as well as administrators involved in the overall allocation of resources and priority setting, we asked each group of two participants from each institution to complete the survey together. This approach sought to stimulate conversation between institutional participants as well as to hopefully encourage them to reach consensus on the characteristics that each institutions would use to describe itself. The survey consists of 13 Likert-scale questions and 4 open-ended questions. The Likert-scale questions and a summary of the responses of the 16 institutions participating in the workshop are shown in Table 1 (the numerical values correspond to 1-Strongly Disagree, 2-Disagree, 3-Neutral, 4-Agree, and 5Strongly Agree):

Table 1. NSF Pre-Workshop Survey Questions

1. Our faculty members are encouraged to be mentors to undergraduates.

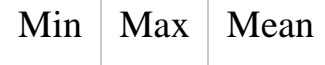

2. Our faculty often build lasting relationships with undergraduates that extend well past graduation.

3. Our institution emphasizes experiential learning opportunities.

4. Our institution requires a co-op experience or industry linked project of all undergraduate engineering students.

5. Faculty members are encouraged to do research with undergraduates.

6. High quality undergraduate teaching is essential in our retention, tenure, and promotion process.

\begin{tabular}{|l|l|l|}
\hline 1 & 5 & 4.2 \\
\hline 2 & 5 & 4.3 \\
\hline 3 & 5 & 4.6 \\
\hline 1 & 5 & 2.4 \\
\hline 3 & 5 & 4.3 \\
\hline 2 & 5 & 3.9 \\
\hline 2 & 5 & 3.4 \\
\hline 2 & 5 & 3.6 \\
\hline 2 & 5 & 3.8 \\
\hline 1 & 5 & 3.4 \\
\hline 2 & 5 & 3.8 \\
\hline
\end{tabular}

7. Helping our students find a career direction that they are passionate about is more important at our institution than simply preparing students to be successful engineers.

8. Our institution emphasizes collaborative teaching and learning.

9. Our institution emphasizes interdisciplinary teaching and learning.

10. Developing an entrepreneurial mindset is an important part of our education of undergraduate engineering students.

11. Developing ethics and empathy for others is an important part of our education of undergraduate engineering students. 
12. Developing an appreciation for the global context of engineering is an

important part of our education of undergraduate engineering students.

13. Engineering education research and innovation is important at my institution.

\begin{tabular}{l|l|l}
2 & 5 & 3.9 \\
\hline 2 & 5 & 3.9
\end{tabular}

In general, the responses to the above questions show significant uniformity in the perceived encouragement for, and reality of, faculty mentoring and forming lasting relationships with undergraduates (14 of 16 responses to question \#1 and 15 of 16 responses to question \#2 were either "agree" or "strongly agree”). The institutions participating in the workshop also placed strong emphasis on experiential learning (15 of 16 responses to question \#3 were "agree” or "strongly agree") although only 5 of 16 responded "agree" or "strongly agree" to requiring a "coop experience or industry linked project” of their students. Faculty encouragement for doing research with undergraduates received strong emphasis, with 15 of 16 respondents agreeing or strongly agreeing. In question \#6, 12 of 16 institutions responded "agree” or "strongly agree” to the statement that high quality teaching is essential in retention, tenure, and promotion. While high quality teaching is essential, emphasizing helping students find a career direction that they are passionate about rather than simply preparing them to be successful engineers is less strongly supported, with only 6 of 16 institutions responding "agree” or "strongly agree.”

Questions \#8 through \#12 focus on a selection of mindsets (attitudes, behaviors, and motivations) that have been increasingly recognized as essential to the success of engineering graduates, perhaps even more so than simply academic achievement [22]. The five mindsets considered in these questions are: a collaborative mindset, an interdisciplinary mindset, an entrepreneurial mindset, an ethical/empathetic mindset, and a global mindset. In general, these mindsets are emphasized to some degree across all institutions participating in the workshop, with $8,9,10,10$, and 11 of the 16 respondents either agreeing or strongly agreeing that "collaborative teaching and learning," "interdisciplinary teaching and learning," "developing an entrepreneurial mindset," "developing ethics and empathy for others,” and "developing an appreciation for the global context of engineering," respectively, are emphasized at their institutions. The area that generated the greatest disagreement in emphasis is the entrepreneurial mindset with 5 of the 16 responses either "disagree” or "strongly disagree."

The last Likert-scale question of the survey asks if "engineering education research and innovation is important." As expected, given the willingness of the participating institutions to be part of the workshop, 11 of 16 responses were either "agree" or "strongly agree" and there was only 1 total response of "disagree” or "strongly disagree." At these institutions participating in the workshop, engineering education research and innovation clearly play an important role.

In addition to the 13 Likert-scale questions, there were 4 open-ended questions asked of the workshop participants. The questions and a summary of responses are given below grouped according to each question:

\section{Which institutional undergraduate educational goals distinguish your institution from others?}

In response to this question, respondents emphasized areas that are common across a number of institutions, such as opportunities for a broad liberal education, project- or problem-based 
learning, industry collaborations and experiential learning, undergraduate research, and global, service, and entrepreneurial learning opportunities. Less commonly identified, although appearing in multiple responses, were access and affordability as well as retention and diversity. Goals that were truly distinguishing included giving students the opportunity to design their own educational experiences, strong support and encouragement for students to cross traditional disciplinary boundaries, an overarching institutional mission to transform engineering education, and a particular institutional emphasis on serving the needs of Hispanic engineering students.

\section{How could participation in engineering education research be increased at your institution?}

In response to this question, respondents' comments fell into a relatively small number of categories. A common and overarching response was simply the need to promote a better understanding of what engineering education research (EER) is and how faculty not directly involved in EER can benefit from that research, with one respondent succinctly stating, "faculty remain unaware of the potential of EER to transform the way we practice engineering education." There was recognition that a large amount of high quality engineering education research has been done over the last couple of decades but its impact is yet to be fully felt in the teaching occurring at most institutions.

Recommendations for increasing the adoption of well-established engineering education research included seed grants (both internal and external) and better administrative support and encouragement for those faculty willing to adapt and change the way they teach. Another suggestion for increasing the awareness and translation of EER into teaching practices was to ensure that each engineering department has a number of faculty members within it that are doing engineering education research and can share their experiences with colleagues. Related directly to increasing the foundational base of EER were suggestions for greater collaborations between departments or schools of education and engineering departments and forming partnerships between research focused institutions and predominantly undergraduate institutions to increase the cross flow of ideas between researchers and practitioners.

Overarching all of these ideas was the recognized need for a fundamental shift in retention/reappointment, tenure, and promotion (RTP) criteria. RTP criteria at most institutions do not generally fully reward engineering education research and the impact of its application, although there was an awareness that changing RTP criteria in significant ways is extremely challenging. Although the number of institutions that have a department of engineering education and offer a Ph.D. degree in engineering education is increasing, another suggestion was to further increase the number of engineering education departments nationwide and to grow the existing ones.

\section{What are the greatest obstacles/opportunities for undergraduate curricular change at your institution?}

The responses to this question focused largely on obstacles, but there were also a number of opportunities that were identified. The obstacles focused on time, resources, ABET, rigid curricular structures, siloed attitudes within and among departments, and faculty mindset, with 
the faculty being characterized as "averse to change and great at seeing the 100 reasons that exist for NOT doing something” as well as having "a very traditional transmissionist view of learning." This faculty resistance to change is not true at all the institutions surveyed, however, with some institutions saying that "change happens all the time" and characterizing themselves as "agile from a perspective of curricular change." Even at institutions at which change is common, there were concerns expressed about the pressures created by success, with one response stating that a "history of success makes stakeholder resistant to change."

A characteristic of institutions that can be both an obstacle and an opportunity is size. Large institutions tend to be bureaucratically rigid but large numbers of students allow for comprehensive explorations of the impact of instructional innovation. Small institutions tend to view themselves as agile but acknowledge that evaluating the impact of change is difficult and "testing new ideas at scale is impossible."

Opportunities for undergraduate curricular change focused on the need for greater recognition of the need for change by actively seeking input from faculty, students, alumni, graduate schools, and employers as well as, as in the responses to question \#2, changes to retention/reappointment, tenure, and promotion criteria. There was recognition that the "tenure and promotion process has not traditionally expected faculty members to make contributions to undergraduate curricular change." Other opportunities for curricular change that were identified included the creation of faculty teams to lead broad-based change initiatives (rather than relying on individual faculty members) and taking advantage of funding opportunities to support change such as National Science Foundation IUSE/PFE: REvolutionizing engineering and computer science Departments (IUSE/PFE: RED) grants.

\section{If you were an NSF program director, what major engineering education research project would you propose and champion?}

Below is a sampling of specific suggestions for funding initiatives suggested by workshop participants, which focused on creating or enhancing NSF support for:

- Infusing more design thinking into engineering education

- Assessing best practices nationwide and methodologies for assessment

- Placing engineering education graduate students (pre-docs, post-docs) in institutions

- Implementing engineering education research and creating ways "for getting faculty to adopt practices that we already know are more effective"

- "Systematic Change: Working up the ladder (or down the iceberg) As an example, most engineering programs espouse to value collaboration and teaming among their students, but how do collaboration and teaming translate 'up the ladder'? How many engineering deans are making room in their schools for faculty to collaborate on course or curriculum design, or provide reasonable support for team-based teaching? How many faculty promotion and tenure review processes support collaboration and social learning, as opposed to individual performance? How many universities embrace a collaborative (vs. competitive, scarcity) mindset in recruiting faculty and students?”

- New approaches to diversity in engineering 
- Developing alternate evaluation techniques for teaching effectiveness and student learning

- Engineering education research projects outside of undergraduate education

- Programs, such as the Grand Challenge Scholars Program, that "do not follow traditional disciplinary boundaries and that integrate broader contexts" and "get faculty and students involved in real-world interdisciplinary design projects?”

- Making "high-quality training in how people learn (the cognitive, social, cultural, and emotional factors of learning)" part of all NSF graduate research fellowships

- Programs that demonstrate "ways to develop broader skills/attitudes while simultaneously improving technical outcomes"

- Programs that study methodologies for developing within students the mindsets that promote "more holistic systems level understanding that connect what engineering learners need to learn, and what attitudes and beliefs are desired"

\section{Workshop Structure and Preliminary Outcomes}

The overarching goal of the workshop was to enhance conversion of engineering education research into practice and to promote collaboration among institutions of varying size and mission. The workshop agenda was specifically designed: i) to provide actionable guidance to the National Science Foundation for future research in engineering education and ii) to catalyze innovation in engineering education through workshop generated interactions and ideas.

The workshop was structured such that participants had extensive opportunities to interact with each other, to learn from each other's experiences and identify common interests, and to build relationships with the potential to create pathways to working together to address common challenges, opportunities, and desired outcomes. These goals were achieved during the course of the workshop through a wide variety of activities built around exercises such as "Speed Networking," "Discovery," and "Partnering." The exercises focused on the characteristics that unite and differentiate engineering education at different institutions, and the shared experiences that will help prepare the attendees and their institutions for the educational changes to come in the future.

Beyond active interactions, the participants also heard two presentations that helped to frame the workshop as well as to focus discussions. The workshop began with a talk by Dr. Richard Miller, President of Olin College, who gave an overview of the "State of Engineering Education" and in particular highlighted the evolving trend in engineering education away from preparing students for the "Knowledge Economy" to preparing them for the "Innovation Economy." Dr. Miller emphasized the importance of mindsets in engineering education and the cultivation of attitudes, behaviors and motivations. He specifically talked about the need to develop within students a Collaborative Mindset, an Entrepreneurial Mindset, an Interdisciplinary Mindset, a Global Mindset, and an Interdisciplinary Mindset. Dr. Elliot Douglas, Program Director within Engineering Education Research at NSF gave participants a deeper understanding of the goals of the workshop and in particular focused on the need to move beyond simply more engineering education research and to develop more effective paths to implementation of well understood and effective engineering pedagogies. Dr. Douglas also emphasized the need to engage a broader range of institutions in cultivating change in engineering education, particularly 
institutions that educate large numbers of engineering students but for a variety of reasons do not typically seek NSF funding.

The Speed Networking exercise gave participants an opportunity to get to know each other better and to share in brief, 5-minute, one-on-one conversations their broad experiences in engineering education at their home institutions. Conversations occurred within administrator-toadministrator and faculty-to-faculty groups in round one, and in mixed groups of administrators and faculty in round two. Conversations were wide ranging and touched upon topics such as the gender gap in engineering education, growing enrollments, students coming from underrepresented groups, the "culture" of engineering, "real-world” projects, new approaches to teaching, faculty evaluation and reward systems, programmatic details, impediments to change, the challenge of the sophomore year student experience, creating cross-disciplinary opportunities, administrative bureaucracy, "this-is-how-I-learned-it” mentality, and many others.

Following the networking activity was a Discovery exercise that was broken into two parts. The first sought to identify the knowledge and experiences 1) that each individual institution was best prepared to share with other institutions and 2) that they were most in need of learning more about. Knowledge and experiences that institutions were best prepared to share included:

- Converting engineering educational research into practice

- Living without departments and promoting interdisciplinarity

- Curricula structured around design thinking and human-centered engineering

- Approaches to promoting diversity and inclusion

- Deep experience with project-based learning and promoting international experiences

- Being a field site for engineering education research

- Technology to promote social/collaborative learning

- Developing external project sponsors

Areas in which institutions were most in need of learning more included:

- Developing programs fostering diversity and inclusion

- Encouraging student passion/motivation

- Maximizing individual students' experiences

- Cultivating shared values among faculty

- Incentivizing a culture of change and teaching excellence

- Assessment of student learning

- Designing more effective learning spaces

- Fostering high impact experiences

- Creating engineering experiences for liberal arts students

The second part of the exercise asked participants to review the "share" and "learn" items posted by each institution around the room and to then place sticky notes on the items to which their institution would most be interested in contributing. With further general discussion, there emerged 5 overarching themes that seemed to capture the areas in which participants felt there was the most interest and opportunity: 
- Diversity and Inclusion

- Engineering Culture and Identity

- Community and Collaboration

- Assessment

- Teaching Excellence

After lunch, participants returned to these 5 themes and were asked to form groups that could brainstorm potential research areas within each. Each of the groups rotated through the themes and made comments so that each participant had the opportunity to contribute their thoughts on each of the 5 themes. The groups added research questions or potential areas of investigation. A very large number of ideas were generated, but research questions and areas of investigation that seemed to be of particular interest within each theme were:

- Diversity and Inclusion:

o Beyond recruitment, how can we sustain, support, and help to thrive a diverse population of students?

o How can we cultivate empathy among faculty and students and how would that improve diversity and inclusion?

o How do we create an understanding for non-diverse populations of the value of diversity?

- $\quad$ Engineering Culture and Identity

o How can/should engineers work with anthropologists and sociologists to better understand cultural identity?

o How can we encourage students to develop an engineering mindset that goes beyond technical?

o How do instructors shape the mindset of engineering students and how can we be intentional in that shaping?

- Community and Collaboration

o What structures and frameworks are most productive in cultivating interactions between engineering education practitioners and researchers?

o How can we more effectively disseminate to practitioners the best practices identified through engineering education research?

o How can we create broader acceptance of retention/reappointment, tenure, and promotion criteria that are more supportive of engineering education research?

- Assessment

o How can we develop alternative approaches to the evaluation of faculty for retention/reappointment, tenure, and promotion that better link to teaching effectiveness, student learning outcomes, and cultural change?

o How can we develop truly scalable assessment techniques for evaluating student work that preserve the strengths of approaches such as rubrics and portfolios?

o How do we assess and enhance developing a growth (versus fixed) mindset in both faculty and students?

- Teaching Excellence

o How do the many aspects of achieving teaching excellence, such as establishing the appropriate learning environment, reacting productively to assessment data, 
noticing and responding to student thinking, and attending to all learners, foster learning?

o How does "excellent" teaching scale with class size; for example, what are the most effective teaching methods for classes less than 15, 15-30, 30-50, and greater than 50 students?

o How do we encourage and reward teaching beyond the classroom and in fact all the diverse ways that learning is accomplished?

Attendees also identified several questions that did not easily fit into the 5 themes but that nonetheless seemed worthy of further consideration, such as

- How can we incentivize institutions to participate in collaborative research?

- How can we get students to de-compartmentalize their learning?

- In what ways does Ph.D. education need to change to be able to adapt and utilize best practices in engineering education as new members of the engineering faculty?"

There were obviously many diverse directions for exploration, but in order to hone the discussion, participants were next asked to work in groups of 3 to 6 to delve more deeply into specific questions and try to answer three fundamental aspects of them:

- What is known?

- What resources are needed?

- What needs to be studied?

The wide range of specific approaches to addressing the many research questions considered is difficult to summarize coherently in the space available here but did very much serve to illustrate the potential for deep and further study in a number of areas. The overall success of this exercise helps to make the case for a comprehensive research agenda worthy of NSF support with the potential for far reaching impacts.

One very important question arose during the workshop in response to a statement about the many extensive discussions, programs, and studies of change in engineering education that have already occurred over the last number of years. That question was, "Are we actually making any progress?" This question addressed the core purpose of the workshop and raised the issue of the true opportunity for change. The workshop organizers considered this question and decided that an unplanned reflective exercise would be valuable using the simple prompt, "What progress have we made?” Each group of two representatives from the attending institutions was asked to reflect and comment on the progress made in the areas of the 5 themes identified earlier in the workshop at either their home institution or nationally within the engineering education landscape.

Responses to this simple prompt were illuminating and in many ways inspiring. While there is clearly much, much more work to be done, a lot has been accomplished within the span of the careers of the attendees. Some of the notable changes include:

- Diversity and Inclusion 
o Significant overall increase in the percentage of women in engineering (some degree programs and even some institutions are graduating more women than men) and other under-represented groups of students and faculty

o Acknowledged need for greater outreach and support of under-represented groups (such as NSF ADVANCE and ESTEEM grants)

o Increased social and global awareness in courses

- Engineering Culture and Identity

0 Broader range of majors and more industrial involvement in projects and CO-OPs

o Teaching of courses specifically focused on culture and identity

o Much greater emphasis on project based learning and learning in teams

o Integrated educational experiences connecting engineering, design, leadership, business, and the arts

o More human centered design opportunities

o Much greater emphasis on innovation and design thinking

o More opportunities for service based learning and engineering for social good

o Greater focus on impact and mindsets

o NSF RED grants driving cultural change

o Study abroad common and expected

- Community and Collaboration

o Engineering education has become a recognized discipline, with departments of engineering education arising nationwide

o National collaborations through programs such as KEEN, the Grand Challenge Scholars Program, and the Maker Movement

o More opportunities and expectations for collaborative teaching

o Collaborations among engineering, business, medicine, public policy, and education programs

o More fully integrated engineering curricula

o Living, learning communities now common

- Assessment

o ABET 2000 and more outcomes based assessment

o Ever more strongly rooted culture of assessment

o Greater use of "big data" to track and assess students

- Teaching and learning centers are expected parts of campuses

0 Growing use of portfolio based assessment

o Courses often structured around Student Learning Outcomes

o Shifts in retention/reappointment, tenure, and promotion criteria to reflect more refined assessments of teaching

- Teaching Excellence

o Much greater awareness of effective teaching practices

o Growth in the "studio" model of engineering education

o Much greater emphasis on high impact experiences and active learning

o Opportunities for teaching preparation now common in many Ph.D. programs

o Greater opportunities for connecting fundamental math and science instruction with engineering principles and project based learning

o Endowed chairs for teaching innovation and teaching excellence 
Engineering education is clearly in a period of change, which participants in the workshop hope is accelerating and can build on many of the advances achieved to date.

One important aspect of the workshop was the opportunity to explore the creation of institutional collaborations and partnerships, although attendees recognized that there are many barriers to building successful collaborations. To consider the challenges of partnering between institutions, particularly those less deeply involved in engineering education research than many of the attendees, one of the last sessions of the workshop created groups to answer the question, "What would it take for each of us to build successful partnerships?" For this exercise, representatives of the following schools were grouped together:

- Arizona State University and Western Kentucky University

- Olin College and Ohio State University

- Bucknell University, the University of Texas, El Paso, and Purdue University

- Dartmouth College and Oregon State University

- University of San Diego and Stanford University

- Worcester Polytechnic Institute and Harvey Mudd College

- Tufts University and Rose-Hulman Institute of Technology

Responses to the prompt included:

- Personal relationships between individuals at the partnering institutions who recognize the value of the partnership and view it as a win-win

- Necessary resources to engage in the partnership and implement its goals (especially time, money, facilities, etc.)

- High level administrative support beyond just funding opportunities that includes recognition through institutional retention/reappointment, tenure, and promotion criteria

- Identification of entrepreneurially minded faculty who are recognized as successful in developing collaborative projects to serve as champions and role models

- Funding through national agencies such as NSF, particularly for institutions that typically do not participate in NSF funding opportunities

- Demonstrated likelihood of sustainability beyond initial funding and partnership creation

- Opportunities to use partners as field sites

- Opportunities to disseminate results and highly impact others

- Direct engagement of students and teaching assistants in the creation of partnerships

- Adjustment of teaching loads to recognize the efforts needed to build partnerships

- Structuring of partnerships between groups of faculty not just individuals

- Creation of a "concept warehouse” to provide a clearinghouse for identifying and building partnerships

- Inclusion of sustainability, and potentially sunsetting, as part of partnership planning

- Using differences between institutions as strengths to collaboration

- Ensuring partnerships are not dominated by a single or small group of institutions; a balanced partnership tailors goals differently for different participating institutions

- Involving disciplines beyond just engineering in the partnership 
The last exercise of the workshop returned to its central purpose of catalyzing a research agenda for the National Science Foundation for enhancing engineering education. Participants were asked specifically what message from the workshop should be communicated to NSF.

Responses included:

- NSF must be willing to help fund the infrastructure need to successfully submit and enact proposals, particularly for those institutions that have historically not received funding

- Partnerships around engineering education can be built upon structures already in place for other NSF-funded collaborations

- Support should avoid "The Hero Engineer" syndrome in which one institution imposes its views and successes on another; true listening and dialogue must occur

- Truly successful approaches to change in engineering education must be recognized and made available to others, perhaps through a clearinghouse structure

- To have impact NSF must be willing to go beyond research and support development

- Structures must be created to truly reward collaborations not individuals

- NSF should be collaborating with other organizations to help bring about change

- NSF should be making more support available at the Ph.D. level to start the process of changing mindsets and experiences before students become faculty

- NSF should study institutions that are not successful at getting awards and writing proposals and investigate why they are "not showing up"

- Creativity/innovation should be rewarded in the same way as research/success

- Change in engineering education is cultural change not curricular reform; partnerships must be built with those studying culture, such as anthropologists and sociologists

The workshop concluded with an evaluation of the experience for the participants. Overall, the workshop was viewed very favorably with ratings of 4.7 out of 5 , with 1 being "disagree strongly" and 5 being "agree strongly" in response to the statement "The workshop was a valuable experience." Similar responses were received for "The workshop was enjoyable" (4.8), "The workshop will impact my future activities" (4.4), and "The overall workshop was excellent” (4.6). Individual activities were also viewed favorably with the opportunities to network and share ideas being described as particularly valuable. One participant commented that the most valuable aspect of the workshop was, "The opportunity to meet faculty and administrators from other institutions and collectively map out the most important issues and research opportunities in engineering education today.” Suggestions for improvements included logistics (better shuttle service, food, and space layout) as well as more significant comments related to more time to explore ideas, delve more deeply into specifics, build structured partnerships, and reach a definitive concluding recommendation.

A specific recommendation made by one of the participants was to engage Prof. Gary Downey in the Department of Science and Technology in Society at Virginia Tech. Prof. Downey is a cultural anthropologist who studies the culture(s) of engineering. Since one of the comments that was heard repeatedly throughout the workshop was the need to focus on culture rather than content, the workshop co-organizers reached out to Prof. Downey to seek his observations on the current and evolving culture within the profession of engineering and within engineering education. In a lengthy and illuminating conversation with Prof. Downey, he described the need to design courses that encourage students to reflect on knowledge, identity, and commitment, with 
the need for reflection being critical. He pointed out that engineers in different countries define problems differently based on the infrastructures of engineering formation and work, through which they pass and to which they contribute. He stated that there is a fallacy in expecting engineering education research to influence traditional approaches to education "based on rational arguments." He also stressed the need to avoid engineers becoming viewed merely as technicians, particularly when working in interdisciplinary groups, and to develop a model of engineering as collaborative Problem Definition and Solution (PDS) [24]. An interpretation of this is that we must avoid the commoditization of engineering and ensure that our students' educational experiences provide more than just content knowledge and instill the mindsets and broad outlooks they will need to be successful in their careers. When asked how to achieve change, Prof. Downey suggested starting with the present rather than focusing solely on the ultimate goals and asking, "What's involved in the next step?"

\section{Summary}

The ultimate goal of our workshop was to create a framework for the development of a more impactful engineering education research agenda that acknowledges the diversity of types and sizes of institutions of higher education. Preliminary steps were taken to generate topics for a research agenda that informs the NSF and the broader stakeholder community and catalyzes more rapid change in engineering education. Data from the workshop, which is still being analyzed, will enable the NSF to identify an actionable research program for creating productive collaborations that support strategies having demonstrated potential for sustainable change.

The specific outcomes that have been achieved or are currently being generated through the workshop include:

- compilation of proposed curricular or pedagogical initiatives, experiments, and potential outcomes identified by participants;

- collection of data at participating institutions documenting previous practices and new initiatives with measures of learning outcomes;

- $\quad$ analysis of collected data to identify trends and areas for future study;

- development of pre- and post-implementation assessment tools based on observations and workshop discussions.

As a result of this workshop, the need became clear for the National Science Foundation to be willing to support the development and implementation of advances in engineering education rather just research into engineering education. There was also a fundamental challenge identified for pursuing support from NSF at schools for which institutional support, recognition, and time for grant related activities is limited. In fact, at some institutions at which NSF hopes to expand engagement in engineering education research, the potential negative impact of writing a funded proposal may be as high or higher than writing an unfunded proposal. In both cases, the investment of time and effort is significant, and in the case of the funded proposal, the bandwidth and support for achieving the goals of the proposal may be severely limited by competing institutional demands. Overall, institutions of higher learning, the NSF, and the engineering professional societies have not succeeded in creating the right culture, climate, and educational infrastructure for implementing change in engineering education based on the most effective 
approaches to engaging students. Traditional engineering education has focused on technical problem definition. We need to transform engineering education so that it focuses on defining problems differently and expands the base of engineering education and the students who are attracted to it.

To change the focus of engineering education requires a shift in the mindsets of faculty and the mindsets they cultivate in their students. Institutions must work together to do this, and this workshop has hopefully planted the seed for the creation of a functional "network of networks" of institutions. Each of the institutions invited to the workshop has their own stakeholder communities that support their goals and efforts in engineering education. This workshop created an opportunity to bring together a diverse range of institutions and begin a process of productive communication and collaboration among them. Ultimately, the workshop will hopefully play a significant role in identifying and shaping a new global agenda for engineering curricula.

\section{Acknowledgements}

The authors gratefully acknowledge the efforts and engagement of the workshop participants. In addition, the authors thank the National Science Foundation (NSF) for support of this research (award EEC-1654206). The views expressed herein are solely those of the authors'.

\section{References}

[1] Lightman, A. and Sadler, P. "Teacher predictions versus actual student gains,” The Physics Teacher, 31, 162, 1993.

[2] Suping, S. “Conceptual change among students in science.” ERIC Digest ED482723, 2003.

[3] Streveler, R. T., Litzinger, T., Miller, R., and Steif, P., "Learning conceptual knowledge in the engineering sciences: Overview and future research directions,” Journal of Engineering Education, 97(3), 279-294, 2008.

[4] Prince, M.J., Vigeant, M., and Nottis, K., “Assessing misconceptions of undergraduate engineering students in the thermal sciences,” International Journal of Engineering Education 26(4), 880-890, 2010.

[5] Prince, M.J., Vigeant, M., and Nottis, K., “Assessing the prevalence and persistence of engineering students' misconceptions in heat transfer,” Journal of Engineering Education 101(3), 412-438, 2012.

[6] National Research Council, "Discipline-based Education Research: Understanding and Improving Learning in Undergraduate Science and Engineering,” S.R. Singer, N.R. Nielsen, and H.A.

Schweingruber (eds.), Washington, D.C., 2012.

[7] Freeman, S., Eddy, S.L., McDonough, M., Smith, M.K., Okoroafor, N., Jordt, H., and Wenderoth, P.M., "Active learning increases student performance in science, engineering, and mathematics," Proceedings of the National Academy of Sciences of the United States of America, 111(23), 8410-8415, 2014.

[8] Deslauriers, L., Schelew E., and Wieman, C., "Improved Learning in a Large-Enrollment Physics Class,” Science, 332(6031), 862-864, 2011. 
[9] Haak, D.C., HilleRisLambers, J., Pitre, E., and Freeman, S., "Increased Structure and Active Learning Reduce the Achievement Gap in Introductory Biology,” Science, 332(6034), 1213-1216, 2011.

[10] Prince, M.J. and R. Felder, "Inductive Teaching and Learning Methods: Definitions, Comparisons, and Research Bases”, Journal of Engineering Education, 95(2), 123-138, 2006.

[11] Prince, M., “Does Active Learning Work? A Review of the Research”, Journal of Engineering Education, 93(3), 2004.

[12] Morton, C.S., Huang-Saad, A., and Libarkin, J., "Entrepreneurship Education for Women in Engineering: A systematic review of entrepreneurship assessment literature with a focus on gender," Proceedings of the ASEE Annual Conference and Exposition, 2016.

[13] Gerhart, A.L. and Melton, D.E., "Entrepreneurially Minded Learning: Incorporating Stakeholders, Discovery, Opportunity Identification, and Value Creation into Problem-based Learning Modules with Examples and Assessment Specific to Fluid Mechanics," Proceedings of the ASEE Annual Conference and Exposition, 2016.

[14] Kirkpatrick, S.R., Watt, A., and Bernal, A., "Developing an Entrepreneurial Mindset in Engineers: An Application of the Three C's (Creativity, Curiosity and Connections) in a Collaborative Summer Mega-course,” Proceedings of the ASEE Annual Conference and Exposition, 2016.

[15] National Research Council, Expanding Underrepresented Minority Participation: America's Science and Technology Talent at the Crossroads. 2011, National Academies Press: Washington, DC.

[16] Koller, D., “What We're Learning from Online Education,” TEDGlobal 2012, https://www.ted.com/talks/daphne_koller_what_we_re_learning_from_online_education?language=en, June, 2012.

[17] Busteed, B., "Higher Education’s Work Preparation

Paradox,” http://www.gallup.com/opinion/gallup/173249/higher-education-work-preparationparadox.aspx, 2014.

[18] Reed-Rhoads, T., Imbrie, P.K., Haghighi, K., Radcliffe, D., Brophy, S., Ohland, M. and Holloway, E., "Creating the Ideas to Innovation learning laboratory: A first-year experience based on research,” International Journal of Engineering Education, 26(5), 1-14, 2010.

[19] ASEE Engineering College Profiles and Statistics, https://www.asee.org/papers-andpublications/publications/college-profiles, 2015.

[20] U.S. News \& World Report Best Colleges, http://colleges.usnews.rankingsandreviews.com/bestcolleges, 2017.

[21] Integrated Postsecondary Education Data System (IPEDS), U.S. Department of Education, Institute of Education Sciences, National Center for Education Statistics, https://nces.ed.gov/ipeds/, 2014, 2015.

[22] Miller, R.K., “The Importance of Mindset,” http://olin.edu/sites/default/files/oct_16_white_paperthe_importance_of_mindset1.pdf, 2016. 
[23] The New York Times Economic Diversity and Student Outcomes Interactive Tool, https://www.nytimes.com/interactive/projects/college-mobility, 2017.

[24] Downey, G., “Are Engineers Losing Control of Technology? From 'Problem Solving' to 'Problem Definition and Solution’ in Engineering Education,” Chemical Engineering Research and Design, 83(A6), 583 - 595, 2005. 